SHORT REPORT

\title{
Associations between internet sex seeking and STI associated risk behaviours among men who have sex with men
}

\author{
A Mettey, R Crosby, R J DiClemente, D R Holtgrave
}

Sex Transm Infect 2003;79:466-468

Objective: This exploratory study identified associations between internet sex seeking and HIV associated risk behaviours among a high risk sample of men who have sex with men (MSM).

Methods: A cross sectional survey of men attending a sex resort was conducted. Of 164 men asked to participate, 91\% completed a self administered questionnaire. The questionnaire assessed demographic variables and (using a 3 month recall period) men's HIV associated sexual risk behaviours. Potential confounding variables were assessed and controlled, as needed, by multivariate analysis.

Results: Men currently resided in 14 states. One sixth reported being HIV positive. $57 \%$ of the men reported using the internet to seek sex. Differences in critical behaviours (unprotected anal sex and number of partners) were not found. However, compared to those not seeking sex by internet, men using the internet to meet sex partners were more likely to report fisting (adjusted odds ratio $=3.3$, $p=0.04$ ), having group sex (prevalence ratio $(P R)=1.2$, $p=0.0001)$, using poppers during sex $(P R=1.94$, $p=0.0001)$, and using ecstasy during sex $(P R=2.7$, $p=0.04$ ). Internet sex seeking men were also significantly more likely to report meeting sex partners in bathhouses $(P R=2.2, p=0.0001)$, bars $(P R=1.5, p=0.001)$, parks $(P R=3.2, \quad p=0.006)$, and circuit parties $(P R=8.9$, $\mathrm{p}=0.007)$

Conclusion: Among MSM attending a sex resort, those using the internet to seek sex partners may have modestly elevated risks for acquiring or transmitting sexually transmitted infections. Subsequent studies should investigate the utility of using the internet as forum for promoting safer sex behaviours among high risk MSM.

$\mathrm{T}$ he internet has become a venue for seeking sex partners. $^{1-5}$ Thus, exploratory research that examines the potentially inflated risk of STI acquisition or transmission among those who use the internet for sex seeking is warranted-for example, recent research has demonstrated the popularity of internet sex seeking among men who have sex with men (MSM). ${ }^{45}$ Some evidence suggests that internet sex seeking may be associated with increased sexually transmitted infection (STI) risk among MSM-for example, one study found that internet sex seeking men reported having a greater number of past STIs compared to those not seeking sex by internet. ${ }^{5}$ Also, an outbreak of syphilis among MSM has been linked to an online chatroom. ${ }^{2}$

Based on surveillance suggesting rising rates of STI infection (including HIV) among $\mathrm{MSM}^{6-10}$ research addressing potential associations between internet sex seeking and STI risk among MSM may be valuable. Accordingly, the purpose of this exploratory study was to identify associations between internet sex seeking and STI associated risk behaviour among a high risk sample of MSM.

\section{METHODS}

\section{Study sample}

During selected Saturdays from May through November 2002, 164 men attending a sex resort located in the southern United States were arbitrarily approached by trained male research staff and asked to participate in a brief survey. Of these men, 150 completed a self administered questionnaire (yielding a response rate of $91 \%$ ). Incentives for participation were not provided. The Emory University institutional review board approved the study protocol before study implementation.

The sex resort was a 65 room complex enclosed by a privacy fence and with a gated entrance. Depending on the season, between 50 and 100 men typically registered at the resort each week. The resort provided men with a steam room, hot tub, maze, dungeon, and an outdoor pool and patio area. Patrons were 18 years of age or older and were admitted to the resort based on membership requirements. Men had the option of renting a room or buying a "day pass" (most patrons rented rooms). In contrast with bathhouses, sex resorts cater for couples and groups of MSM as well as singles. Men may remain at the resort for extended stays (staying in "hotel-like" rooms for one or several days and nights). The environment supported cruising. Clothing was optional in specified areas.

\section{Data collection}

Men were recruited for study participation only during their leisure time spent on the patio or near the pool area. Men providing informed consent were given an anonymous questionnaire that contained 48 closed ended items designed to assess STI associated risk behaviours. Most men took about 15 minutes to complete these items. Men completed the questionnaire at tables within the patio area or near the pool; a few completed the questionnaire in their rented rooms. Men were given a large manila envelope to conceal their completed questionnaire from the researchers.

\section{Measures}

Internet sex seeking

A single item assessed internet sex seeking. Men were asked whether they had ever used the internet for the sole purpose of meeting sex partners.

STI associated risk behaviours

Using a 3 month recall period, frequency of engaging in unprotected anal sex and the number of sex partners was 
assessed. Men were also asked whether they had engaged in the practices of fisting, rimming (a practice especially likely to transmit hepatitis A), and group sex. Men were asked if they had used poppers, ecstasy, whip-its (nitrous oxide), or cocaine during sexual encounters. Finally, men were asked to indicate the venues they had ever used for the sole purpose of meeting sexual partners (that is, baths, bars, parks, public restrooms, circuit parties).

\section{Assessment of potential covariates}

Several measures were assessed as potential confounding variables. Demographic measures (age, race/ethnicity, and income) as well as men's self reported HIV serostatus and history of STI infection were assessed. Whether men were involved with a primary partner (with the relationship lasting at least 1 year) was also assessed.

\section{Data analysis}

Owing to markedly skewed distributions, continuous level outcome measures were dichotomised by performing a median split. Associations between internet sex seeking and the outcome measures were assessed by prevalence ratios, their 95\% confidence intervals, and respective $\mathrm{p}$ values. Outcomes achieving significance $(p \leqslant 0.05)$ were evaluated in hierarchal logistic regression models to control for potential confounding influences of the assessed covariates.

\section{RESULTS}

\section{Characteristics of the sample}

Men residing in 14 states comprised the sample. Average age of the men was 40.7 years (SD 9.4; median 40 years). Most $(93 \%)$ self identified as white. The median annual income interval was \$25 000-\$50 000. One sixth (16.7\%) reported they were HIV positive; $39 \%$ reported ever having an STI other than HIV. Men reported having sex with a mean of 10.0 partners in the past 3 months (SD 42.0; median 4). Just over a half $(51.4 \%)$ of the men reported they were currently involved in a primary relationship. The median length of these relationships was 3-5 years.

\section{Bivariate associations}

Table 1 displays descriptive information and the prevalence ratios corresponding to each bivariate comparison. The descriptive information (shown in the first two columns) provides indicators of STI associated risk, stratified by internet sex seeking-for example, fisting was reported by $22.4 \%$ of the men who reported internet sex seeking compared with $6.8 \%$ among those not seeking sex by internet. This difference was significant, with men seeking sex by internet being 3.3 times more likely to report fisting in the past 3 months. Although engaging in group sex was also significantly associated with internet sex seeking, the prevalence ratio was quite small (less than 1.2). The use of two substances was significantly related to internet sex seeking: poppers and ecstasy. Also, men seeking sex by internet were more likely to meet sex partners in each of the assessed venues (although the association with public restrooms was only marginally significant).

\section{Multivariate associations}

Income was the only assessed covariate that was significantly associated with both internet sex seeking and one of the assessed outcomes (fisting). After adjusting for income, men who used the internet to seek sex were about 3.3 times more likely to report fisting than those who did not use the internet to seek sex (adjusted odds ratio 3.32, 95\% CI 1.04 to 10.57 , $\mathrm{p}=0.04)$.

\section{DISCUSSION}

Findings did not support the hypothesis that internet sex seeking is associated with unprotected anal sex or having a greater number of partners among a sample of MSM attending a sex resort. Prevalence ratios representing associations between internet sex seeking and these measures were very small $(<1.2)$. Although these associations may have achieved significance with a much greater sample size, the small effect size difference has, at best, little appreciable clinical significance.

However, to the extent that fisting, group sex, and the use of poppers and ecstasy magnifies the odds of STI acquisition or transmission, internet sex seeking may indeed be a behavioural marker of greater STI risk. Similarly, to the extent that meeting partners in venues such as parks, bars, and circuit parties magnifies the odds of encountering sex partners infected with an STI, ${ }^{11}$ internet sex seeking may be a proxy of enhanced STI risk.

\begin{tabular}{|c|c|c|c|c|c|}
\hline \multirow[b]{2}{*}{ Outcome measure } & \multicolumn{2}{|c|}{ Internet sex seeking } & \multirow[b]{2}{*}{ PR† } & \multirow[b]{2}{*}{ 95\% Clł } & \multirow[b]{2}{*}{ p Value } \\
\hline & Yes (\%) & No (\%) & & & \\
\hline \multicolumn{6}{|l|}{ Risky sex* } \\
\hline Any unprotected anal sex & 53.0 & 45.8 & 1.16 & $0.82-1.63$ & 0.39 \\
\hline Greater number of sex partners & 53.0 & 44.8 & 1.18 & $0.83-1.68$ & 0.34 \\
\hline Fisting & 22.4 & 6.8 & 3.30 & $1.18-9.20$ & 0.01 \\
\hline Rimming & 66.3 & 57.6 & 1.15 & $0.88-1.50$ & 0.29 \\
\hline \multicolumn{6}{|c|}{ Substance use during sexual encounters* } \\
\hline Poppers & 62.4 & 32.2 & 1.94 & $1.29-2.90$ & 0.0001 \\
\hline Ecstasyt† & 19.3 & 7.0 & 2.75 & $0.97-7.79$ & 0.050 \\
\hline Whip-its (nitrous oxide) & 8.3 & 1.7 & 4.83 & $0.61-38.24$ & 0.09 \\
\hline Cocaine & 16.7 & 8.5 & 1.97 & $0.75-5.16$ & 0.15 \\
\hline \multicolumn{6}{|l|}{ Sexual venue } \\
\hline Baths & 53.5 & 23.7 & 2.25 & $1.37-3.71$ & 0.0001 \\
\hline Bars & 76.7 & 50.8 & 1.51 & $1.15-1.99$ & 0.001 \\
\hline Parks & 26.7 & 8.5 & 3.16 & $1.27-7.83$ & 0.006 \\
\hline Public restrooms & 22.1 & 10.2 & 2.17 & $0.92-5.11$ & 0.06 \\
\hline Circuit parties & 15.1 & 1.7 & 8.92 & $1.20-66.35$ & 0.007 \\
\hline
\end{tabular}




\section{Limitations}

Findings are limited by the inherent limitations of a cross sectional study design and a convenience sample. The use of multiple comparisons is also problematic given the inflated risk of finding significant associations by chance. The validity of men's responses to the questionnaire is also an important limitation. Further, the study is limited by the low statistical power that is inevitable given a small sample size (nonetheless, the use of a small sample provides assurance that significant effects represent substantially large effect sizes). In addition, men were only asked about internet use for the sole purpose of meeting sexual partners, they were not asked whether sex occurred with partners met online. Indeed, the use of a single item to assess internet sex seeking may be problematic; subsequent studies should measure this behaviour in greater detail. Finally, it should be noted that outcomes assessed over a 3 month recall period-for example, frequency of unprotected anal sex and number of sex partners-may have occurred long after men last engaged in sex seeking. Clearly, studies that establish a temporal relation between internet sex seeking and STI associated risk behaviours are needed.

\section{Conclusions}

In analyses accounting for confounding variables, findings suggest that internet sex seeking may not be associated with having unprotected anal sex or having relatively greater numbers of sex partners among MSM attending a sex resort. However, internet sex seeking MSM may have a modestly enhanced risk of STI acquisition (and transmission) by their increased likelihood of fisting, engaging in group sex, use of substances, and meeting sex partners in multiple venues. Subsequent studies should investigate the utility of using the internet as forum for promoting safer sex behaviours among high risk MSM.

\section{Authors' affiliations}

A Mettey, R Crosby, R J DiClemente, D R Holtgrave, Rollins School of
Public Health, Department of Behavioral Sciences and Health, Education, Atlanta, GA 30322, USA

R Crosby, R J DiClemente, D R Holtgrave, Emory Center for AIDS Research, Atlanta, GA 30322, USA

R J DiClemente, Emory University School of Medicine, Department of Medicine (Infectious Diseases), and Emory University School of Medicine, Department of Pediatrics, Atlanta, GA 30322, USA

Correspondence to: Richard Crosby, PhD, Rollins School of Public Health of Emory University, Department of Behavioral Sciences and Health Education, 1518 Clifton Road, NE, Room 542, Atlanta,

GA 30322, USA; rcrosby@sph.emory.edu

Accepted for publication 2 July 2003

\section{REFERENCES}

1 Chiasson M, Hirschfield S, Humberstone M, et al. The internet and high-risk sex among men who have sex with men [oral abstract]. 10th Conference on Retroviruses and Opportunistic Infections 2003;8:37.

2 Klausner J, Wolf W, Fischer-Ponce L, et al. Tracing a syphilis outbreak through cyberspace. JAMA 2000;284:447-9.

3 Elford J, Bolding G, Sherr L. Seeking sex on the internet and sexual risk behaviour among gay men using London gyms. AIDS 2001;15:1409-15.

4 Rietmeijer CA, Bull S, McFarlane M, et al. Risks and benefits of the internet for populations at risk for sexually transmitted infections (STIs): results of an STI clinic survey. Sex Transm Dis 2003;30:15-19.

5 McFarlane M, Bull S, Rietmeijer CA. The internet as a newly emerging risk environment for sexually transmitted diseases. JAMA 2000;284:443-6.

6 Rietmeijer CA, Judson FN, Douglas JM, et al. Increasing rates of gonorrhoea (GC) and other sexually transmitted infections (STI) among men who have sex with men (MSM) compared to men who have sex with women (MSW) in the Denver Metro Health Clinic (DMHC): a 10-year trend analysis. Int J STD AIDS $2001 ; 12: 77$.

7 Centers for Disease Control and Prevention. Gonorrhoea among men who have sex with men-selected sexually transmitted disease clinics, 1993-1996. Morb Mortal Wkly Rep 1997;46:889-92.

8 Centers for Disease Control and Prevention. Outbreak of syphilis among men who have sex with men-Southern California, 2000. Morb Mortal Wkly Rep 2001;50:117-20

9 Centers for Disease Control and Prevention. Need for sustained HIV prevention among men who have sex with men. Available online at www.cdc.gov/hiv/pubs/facts/msm.htm. Accessed 19 November 2002.

10 Sullivan PS, Chu SY, Fleming PL. et al. Changes in AIDS incidence for men who have sex with men, United States 1990-1995. AIDS 1997;11:1641-6.

11 Binson D, Michaels S, Stall R, et al. Prevalence and social distribution of men who have sex with men: United States and its urban centers. J Sex Res 1995;32:245-54.

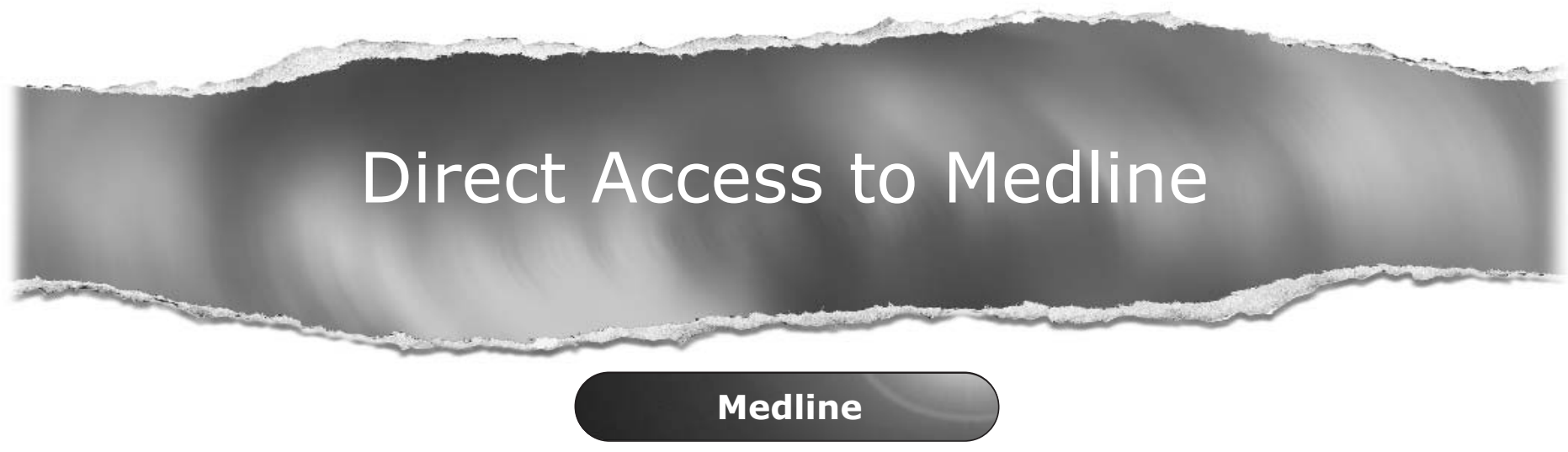

Link to Medline from the homepage and get straight into the National Library of Medicine's premier bibliographic database. Medline allows you to search across 9 million records of bibliographic citations and authors abstracts from approximately 3,900 current biomedical journals. 